PROCEEDINGS OF THE

AMERICAN MATHEMATICAL SOCIETY

Volume 137, Number 11, November 2009, Pages 3943-3949

S 0002-9939(09)09986-9

Article electronically published on June 25, 2009

\title{
CONSTRUCTING NON-CONGRUENCE SUBGROUPS OF FLEXIBLE HYPERBOLIC 3-MANIFOLD GROUPS
}

\author{
D. COOPER, D. D. LONG, AND M. THISTLETHWAITE
}

(Communicated by Daniel Ruberman)

\begin{abstract}
We give an explicit construction for non-congruence subgroups in the fundamental group of a flexible hyperbolic 3-manifold.
\end{abstract}

\section{INTRODUCTION}

When $M$ is a closed orientable hyperbolic 3-manifold, the hyperbolic structure gives rise to a canonical discrete faithful representation of its fundamental group $\rho: \pi_{1}(M) \longrightarrow S O(3,1)$, unique up to conjugacy by Mostow rigidity. Denoting the image group by $\Gamma$, one sees easily from rigidity and finite generation that in fact $\Gamma$ is conjugate to a subgroup of $S O(3,1 ; R)$, where $R$ is obtained from the ring of integers of a number field by inverting a certain finite number of elements.

This situation gives rise to a family of finite representations of the group $\Gamma$ as follows. The ring $R$ is Dedekind, and standard results (see [12], §4.1) imply that for any non-zero ideal $I$, the quotient $R / I$ is finite. Thus the reduction homomorphisms

$$
S O(3,1 ; R) \longrightarrow S O(3,1 ; R / I)
$$

are all finite representations. We say that a subgroup of finite index in $\Gamma$ is a congruence subgroup if it contains the kernel of such a reduction homomorphism and that $\Gamma$ has the congruence subgroup property if every subgroup of finite index is a congruence subgroup; equivalently, the topology on the group coming from the profinite completion is homeomorphic to that coming from the completion using the congruence subgroups.

In his celebrated paper [6, Lubotzky showed that arithmetic hyperbolic 3manifold groups do not have the congruence subgroup property, verifying a conjecture that had originally been made by Serre. However, the method was non-explicit and to the authors' knowledge, no example of a cofinal family of non-congruence subgroups in a closed hyperbolic 3-manifold group has ever been exhibited.

In [3], the authors showed that certain closed hyperbolic 3-manifolds have the property that their hyperbolic structure can actually be non-trivially deformed when considered as a real projective structure. We described such manifolds as flexible. This paper shows that for flexible hyperbolic manifolds, one can explicitly construct a cofinal family of non-congruence subgroups; moreover, since this does

Received by the editors January 7, 2009, and, in revised form, March 31, 2009.

2000 Mathematics Subject Classification. Primary 57M05.

The first author was partially supported by DMS-0706887.

The second author was partially supported by DMS-0706642.

(C)2009 American Mathematical Society Reverts to public domain 28 years from publication 3943 
not rely on [6] in any way, it gives a new proof of the failure of the congruence subgroup property in the flexible case. Furthermore, non-arithmetic flexible manifolds are known [3], so our method applies in cases where Lubotzky's does not. We give a rather explicit construction which shows:

Theorem 1.1. Suppose that $M$ is a closed hyperbolic 3-manifold which is flexible. Then given any non-trivial element $g \in \pi_{1}(M)$, there are infinitely many rational primes $q$ and surjections $\pi_{q}: \pi_{1}(M) \longrightarrow P S L(4, q)$, for which $\pi_{q}(g)$ is non-trivial. Furthermore, $\operatorname{ker} \pi_{q}$ is not a congruence subgroup.

The fact that the kernel of such a homomorphism is not congruence in the sense defined above shows that we have also deduced the failure of the congruence subgroup property for such manifolds.

A (very) special case of a flexible manifold is the situation of a hyperbolic 3manifold which contains an immersed totally geodesic closed surface; it is shown in [3] that such a manifold has a finite cover which is flexible. It follows that:

Corollary 1.2. Suppose that $\Gamma$ is a co-compact Kleinian group which contains a co-compact Fuchsian group. Then the conclusions of Theorem 1.1 hold.

In this more classical form, it seems clear that the result was known to Weisfeiler (see his remarks in the first few paragraphs of $\$ 11$ of [11]). Such manifolds are known which are non-arithmetic, so in this much more restricted setting, Theorem 1.1 was known, at least implicitly.

Here is a sketch of the strategy. The canonical representation of a flexible hyperbolic manifold can be non-trivially deformed when it is regarded as the holonomy of a real projective structure, and this gives rise to nearby convex real projective structures which are faithful discrete representations inside $S L(4, \mathbb{R})$. Deep theorems of Benoist 2] show that the associated deformed holonomies are all Zariski dense subgroups of $S L(4, \mathbb{R})$. We now invoke the Strong Approximation theorem of [11, which implies that the finite representations that one gets by reducing modulo primes in the number field will surject for all but finitely many primes. By choosing a prime which splits completely and which does not kill the image of the prescribed element $g$, we obtain a finite homomorphism which surjects some group $P S L(4, q)$. However the reductions that one obtains by taking the canonical representation and reducing modulo primes will yield image groups which are finite orthogonal groups, and this can be used to show that our homomorphisms are non-congruence.

Finally, we should remark that while flexibility is still very poorly understood, it is known that there are infinitely many commensurability classes of flexible manifolds and one can conjecture that every closed hyperbolic manifold is virtually flexible, so potentially the methods and constructions of this paper are rather general.

\section{Proof of Theorem 1.1}

2.1. Flexible manifolds and Zariski denseness. As is well known, closed hyperbolic manifolds of dimension at least three are rigid, and this implies that the discrete faithful representation $\rho$ of the fundamental group into $O(3,1 ; \mathbb{R})$ is unique up to conjugacy.

However, if one uses the Klein model for hyperbolic space, one can regard this hyperbolic structure as a real projective structure, and as such, it turns out that 
for certain closed hyperbolic 3-manifolds, one can make non-trivial deformations 3. Such manifolds are called flexible.

Theorem 2.1. Let $\rho$ be the discrete faithful representation associated to the hyperbolic structure of a closed hyperbolic flexible 3-manifold.

Then there are arbitrarily small deformations $\rho_{t}$ of $\rho$ which give rise to convex real projective structures. The representations $\rho_{t}$ are faithful and discrete, and the image $\rho_{t}\left(\pi_{1}(M)\right)$ is Zariski dense in $S L(4, \mathbb{R})$.

Proof. It is shown by Koszul [7] that the subset of faithful representations inside $\operatorname{Hom}\left(\pi_{1}(M), S L(4, \mathbb{R})\right)$ for which there is an associated convex real projective structure is open. Thus all the representations $\rho_{t}: \pi_{1}(M) \longrightarrow S L(4, \mathbb{R})$ which are close to $\rho$ are faithful and discrete and induce a convex real projective structure. If $\rho_{t}$ is not conjugate to $\rho$, then this structure is not projectively equivalent to the hyperbolic structure.

The representation $\rho$ is irreducible, and the property of being reducible is a Zariski closed condition, so we may assume that the small deformations under consideration are all irreducible.

We now appeal to Benoist [2, Theorem 1.1, which describes the various possibilities for convex real projective structures in this setting. Since our representations are irreducible, the convex set $C$ associated to the real projective structure which is left invariant by $\rho_{t}\left(\pi_{1}(M)\right)$ cannot split invariantly as a product of convex sets $C_{1} \times C_{2}$.

The statement of Benoist's Theorem 1.1 now becomes either that $\rho_{t}\left(\pi_{1}(M)\right)$ has Zariski closure $S L(4, \mathbb{R})$ or that $C$ is homogeneous; that is to say, $A u t(C)$ acts transitively on $C$. However we may rule out this second case by arguing as follows.

The action of $\operatorname{Aut}(C)$ is by isometries of the Hilbert metric on $C$. Now it is a standard fact (one can base an argument on Theorem 2.17 of [1] for example) that one can associate to any Finsler metric a Riemannian metric which is sufficiently canonical that the action of $A u t(C)$ is by isometries of this Riemannian metric; that is to say, we have assigned to $C$ a Riemannian metric making it into a homogeneous space. This makes $C / \rho_{t}\left(\pi_{1}(M)\right)$ into a closed manifold with a homogeneous metric. Such homogeneous metrics have been classified by Thurston, and the only possibility is that the homogeneous metric on $C / \rho_{t}\left(\pi_{1}(M)\right)$ is a multiple of the hyperbolic metric.

However, we now claim that this contradicts the fact that the representation has been flexed away from the canonical representation. We argue as follows: We have identified $A u t(C)$ as a subgroup of the isometry group of $\mathbb{H}^{3}$ and since the only transitive non-soluble Lie subgroup of $\operatorname{Isom}\left(\mathbb{H}^{3}\right)$ is the whole group, this shows that $\operatorname{Aut}(C) \cong I \operatorname{som}\left(\mathbb{H}^{3}\right)$.

However all representations of $\operatorname{Isom}^{+}\left(\mathbb{H}^{3}\right) \cong S O(3,1)$ into $S L(4, \mathbb{R})$ preserve a non-degenerate invariant bilinear form (see for example [5], p. 205, and Example 3, p. 198). This form cannot be definite since $A u t(C)$ contains an infinite discrete subgroup, and cannot have signature $(2,2)$ since $S O(3,1)$ and $S O(2,2)$ are not locally isomorphic. It follows that we must have signature $(3,1)$ and therefore up to conjugacy in $S L(4, \mathbb{R})$ the representation is equivalent to the standard one. We deduce that the flexed representation can be conjugated into $S O(3,1)$, a contradiction to Mostow rigidity.

We deduce that $C$ cannot be homogeneous and therefore $\rho_{t}\left(\pi_{1}(M)\right)$ is Zariski dense in $S L(4, \mathbb{R}))$, as required. 
2.2. Strong approximation and flexibility. We include here a result that follows from the work of Weisfeiler [11. (Also see Matthews, Vaserstein and Weisfeiler [8] or Nori [9] concerning strong approximation for certain algebraic groups.)

We consider the following situation. Suppose that $\Gamma$ is a finitely generated subgroup of $S L(4, \mathbb{R})$, which has all its entries lying in some number field $k$, so that in fact $\Gamma \leq S L(4, k) \leq S L(4, \mathbb{R})$. Since $\Gamma$ is finitely generated, the entries lie in a subring $R=R_{k}[S]$ of $k$, where $R_{k}$ is the ring of integers of $k$ and a finite number of elements $S$ are inverted. Let $\wp$ be a prime ideal of $R$; then we have a ring homomorphism onto a finite field $\pi_{\wp}: R \longrightarrow R / \wp$ which induces a group homomorphism

$$
\pi_{\wp}: S L(4, R) \longrightarrow S L(4, R / \wp) .
$$

It follows from [1] that for all but finitely many prime ideals $\wp$, this map is actually a surjection.

Applying this in our situation yields:

Proposition 2.2. If $M$ is a flexible closed hyperbolic 3-manifold, then for infinitely many primes $q$ in $\mathbb{Z}$ there is a surjection $\pi_{q}: \pi_{1} M \rightarrow P S L(4, q)$.

Proof. Since $M$ is a flexible hyperbolic 3-manifold it follows from Theorem 2.1 that there is a faithful algebraic specialisation in $\operatorname{Hom}\left(\pi_{1}(M), S L(4, \mathbb{R})\right)$ close to the complete structure for which the holonomy associated to the convex real projective structure is Zariski dense in $S L(4, \mathbb{R})$. By the above, for all but finitely many primes, the reduction homomorphisms are surjective. There are infinitely many rational primes which split completely in $R$, and for ideals $\wp$ lying over such primes, we have that $R / \wp$ is a field of prime order $q$. Now for any prescribed non-trivial element $1 \neq g \in \pi_{1}(M)$, we may choose such a prime ideal $\wp$ in $R$ which does not divide some entry of $\rho_{t}(g)-I$ and thus we obtain a surjective homomorphism

$$
\theta: S L(4, R) \longrightarrow P S L(4, q)
$$

for which the image of $g$ maps non-trivially. Then $\pi_{q}=\theta \circ \rho_{t}$ is the required surjection.

To complete the proof of Theorem 1.1 it only remains to show that $\operatorname{ker} \pi_{q}$ is non-congruence. This will be done in the next section.

2.3. Group theory. In this section, we prove the following, which completes the proof of Theorem 1.1. Let $\Gamma$ be the hyperbolic 3-manifold group and suppose that we have a surjection

$$
\pi_{q}: \Gamma \longrightarrow P S L(4, q)
$$

for some fixed prime $q$. We claim that $\operatorname{ker}\left(\pi_{q}\right)$ cannot be a congruence subgroup.

The following very elementary lemma will be used for the case where $G$ is a direct product and $H$ is one of its factors.

Lemma 2.3. Let $\Sigma$ be a simple group occurring as a subquotient of a group $G$, and let $H$ be a normal subgroup of $G$. Then at least one of $H, G / H$ has a subquotient isomorphic to $\Sigma$.

Proof. By hypothesis we have subgroups $L, M$ of $G$ with $M \triangleleft L$ and $L / M=\Sigma$. Consider first the case where $H \cap L \leq M$. Let $\pi$ denote the natural map from $G$ to $G / H$. Then, since $\operatorname{ker}\left(\left.\pi\right|_{L}\right)=H \cap L$ is contained in $M, \pi$ induces an isomorphism from $L / M$ to $\pi(L) / \pi(M)$, thus exhibiting $\Sigma$ as a subquotient of $G / H$. 
Suppose on the other hand that $H \cap L$ is not a subgroup of $M$. Let $\rho$ denote the natural map from $L$ to the simple group $\Sigma=L / M$. Then $\rho(H \cap L)$ is a non-trivial normal subgroup of $\Sigma$, whence $\rho(H \cap L)$ is the whole of $\Sigma$. Since $\operatorname{ker}\left(\left.\rho\right|_{H \cap L}\right)=H \cap M$, we see that $(H \cap L) /(H \cap M) \cong \Sigma$, exhibiting $\Sigma$ as a subquotient of $H$.

Recall that $\Gamma$ is a subgroup of $S O(3,1 ; R)$, where $R=R_{k}[S]$, meaning $R_{k}$ is the ring of integers of $k$ and there is a finite number of elements $S$ of $R_{k}$ which are inverted. In particular, the ring $R$ is Dedekind. Then if $\operatorname{ker}\left(\pi_{q}\right)$ is congruence, it contains the kernel of a reduction homomorphism

$$
S O(3,1 ; R) \longrightarrow S O(3,1 ; R / I)
$$

for some ideal $I$ of $R$.

Since $R$ is Dedekind, we can factorise the ideal $I$ uniquely as a product of prime ideals $I=\wp_{1}^{k_{1}} \cdots \wp_{r}^{k_{r}}$ and by the Chinese remainder theorem (see [4, Theorem 4, p. 44) we have

$$
S O(3,1 ; R / I) \cong \prod_{j} S O\left(3,1 ; R / \wp_{j}^{k_{j}}\right) .
$$

We denote the images of $\Gamma$ and $\operatorname{ker}\left(\pi_{q}\right)$ in $\prod_{j} S O\left(3,1 ; R / \wp_{j}^{k_{j}}\right)$ by $G$ and $K$ respectively.

Note that any element of the kernel of the map

$$
S O\left(3,1 ; R / \wp_{j}^{k_{j}}\right) \longrightarrow S O\left(3,1 ; R / \wp_{j}\right)
$$

has the form $I+\wp_{j} M$, so has order a power of $p_{j}$ (where $\wp_{j}$ divides the rational prime $p_{j}$ ), and it follows that the kernel of the map

$$
\prod_{j} S O\left(3,1 ; R / \wp_{j}^{k_{j}}\right) \longrightarrow \prod_{j} S O\left(3,1 ; R / \wp_{j}\right)
$$

is a product of $p$-groups and is therefore nilpotent. Denote this kernel by $T$.

Consider the projection map $\mu: G \longrightarrow G / K \cong P S L(4, q)$; the group $\mu(G \cap T)$ is a nilpotent normal subgroup of $P S L(4, q)$, and so is trivial. Therefore $G \cap T \leq K$, so that $K \cap T=G \cap T$.

Let $G^{*}$ and $K^{*}$ be the images of $G$ and $K$ inside $\prod_{j} S O\left(3,1 ; R / \wp_{j}\right)$; let $U$ denote the kernel of the map $G \rightarrow G^{*}$. Then, since $U \leq K$, we have $G^{*} \cong G / U$ and $K^{*} \cong K / U$, whence $G^{*} / K^{*} \cong G / K$. We may therefore reduce to the case that the primes all occur with multiplicity 1 ; that is to say, we have that the product $\prod_{j} S O\left(3,1 ; R / \wp_{j}\right)$ contains a subgroup $G$ with the property that $G$ has quotient $P S L(4, q)$. By repeated applications of Lemma 2.3, we see that we have deduced:

Corollary 2.4. If $\operatorname{ker}\left(\pi_{q}\right)$ is congruence, then for some prime $\wp$, the group $S O(3,1 ; R / \wp)$ contains a subgroup mapping onto $\operatorname{PSL}(4, q)$.

To conclude the argument we need some discussion of the results from the theory of simple groups arising from orthogonal groups which are relevant for us.

We will consider the orthogonal (and special orthogonal) groups of an $m$-dimensional quadratic form over the finite field $\mathbb{F}$ of cardinality $p^{n}$; we shall mostly be concerned with the case $m=4$, and we assume throughout that $p$ is odd. When $m$ is even there are two such orthogonal groups $O_{ \pm}(m, \mathbb{F}$ ) (see [10], p. 377, Theorem 5.8). Let $S O_{ \pm}(m, \mathbb{F})$ denote the special orthogonal group in these cases. 
Let $\Omega_{ \pm}(m, \mathbb{F})=\left[O_{ \pm}(m, \mathbb{F}), O_{ \pm}(m, \mathbb{F})\right]$, where $[G, G]$ denotes the commutator subgroup of a group $G$. When $m$ is even, $\Omega_{ \pm}(m, \mathbb{F})$ has index 2 in $S O_{ \pm}(m, \mathbb{F})$ and has a centre of order 1 or 2 . Let $P \Omega_{ \pm}(m, \mathbb{F})$ be the central quotient group.

We summarize the important facts for us in the following theorem (see [10], pp. 383-384):

Theorem 2.5. When $m=4, P \Omega_{+}(4, \mathbb{F}) \cong P S L(2, \mathbb{F}) \times P S L(2, \mathbb{F})$ and $P \Omega_{-}(4, \mathbb{F})$ $\cong P S L\left(2, \mathbb{F}^{\prime}\right)$, where $\mathbb{F}^{\prime}$ is the finite field of order $p^{2 n}$.

We can now conclude the argument that $\operatorname{ker}\left(\pi_{q}\right)$ is non-congruence. Recall that we have shown that if it is congruence, then for some prime $\wp$, the group $S O(3,1 ; R / \wp)$ contains a subgroup $G$ which maps onto $P S L(4, q)$. As noted above, the commutator subgroup $\Omega_{ \pm}(4, R / \wp)$ has index at most two, so by the simplicity of $P S L(4, q), G \cap \Omega_{ \pm}(4, R / \wp)$ also surjects $P S L(4, q)$. Similarly, we may projectivise and reduce to the case that the domain group is $P \Omega_{ \pm}(4, R / \wp)$.

Using Lemma 2.3 for the case $P \Omega_{+}(4, R / \wp) \cong P S L(2, R / \wp) \times P S L(2, R / \wp)$, we see that Theorem 2.5 now shows that if $\operatorname{ker}\left(\pi_{q}\right)$ is congruence, then for some appropriate finite field $\mathbb{F}, P S L(2, \mathbb{F})$ contains a subgroup mapping onto $P S L(4, q)$. We appeal to [10, Theorem 6.25, page 412, which classifies subgroups of $\operatorname{PSL}(2, \mathbb{F})$ and one sees easily that this is impossible.

2.4. An example. The first flexible manifold in the census turns out to be the manifold usually referred to as vol3. We recall that vol3 (it is the manifold with third-lowest volume in the census) is an arithmetic manifold with volume the same as that of a regular ideal simplex, that is, around 1.01494160640965. The fundamental group has presentation

$$
\langle a, b \mid a a b b A B A b b, a B a B a b a a a b\rangle,
$$

where $A=a^{-1}$ and $B=b^{-1}$. Using the representation of [3], one easily constructs a representation with $q=17$, which $G A P$ computes has order 2851903720876769280; i.e. it surjects $S L(4,17)$ :

$$
\begin{aligned}
& \rho(a)=\left[\begin{array}{cccc}
0 & 0 & 13 & 3 \\
0 & 0 & 12 & 12 \\
1 & 0 & 6 & 5 \\
0 & 1 & 1 & 5
\end{array}\right], \\
& \rho(b)=\left[\begin{array}{cccc}
10 & 0 & 16 & 8 \\
0 & 12 & 1 & 0 \\
0 & 0 & 15 & 6 \\
0 & 0 & 9 & 15
\end{array}\right] .
\end{aligned}
$$

Remark. The first non-arithmetic flexible hyperbolic 3-manifold is $m 34(-4,1)$. One understands the flexing quite explicitly, so using similar ideas one can find a noncongruence representation. However, this computation has not yet been done.

\section{REFERENCES}

1. D. Bao et al. (Editors), A Sampler of Riemann-Finsler Geometry. MSRI Publications, vol. 50. Cambridge University Press (2004). MR2132655 (2005j:53003)

2. Y. Benoist, Convexes divisibles. II. Duke Math. J. 120 (2003), pp. 97-120. MR2010735 (2004m:22018)

3. D. Cooper, D.D. Long and M. Thistlethwaite, Flexing closed hyperbolic manifolds. Geometry \& Topology 11 (2007), pp. 2413-2440. MR.2372851 (2008k:57031) 
4. A. Fröhlich and M. J. Taylor, Algebraic Number Theory, Cambridge Studies in Advanced Math., vol. 27 (1993). MR1215934 (94d:11078)

5. I. M. Gel'fand et al., Representations of the Rotation and Lorentz Groups and Their Applications, MacMillan, 1963.

6. A. Lubotzky, Group presentation, p-adic analytic groups and lattices in $S L(2, \mathbb{C})$, Ann. of Math. (2) 118 (1983), pp. 115-130. MR707163 (85i:22017)

7. J.-L. Koszul, Déformations de connexions localement plates, Ann. Inst. Fourier 18 (1968), pp. 103-114. MR0239529 (39:886)

8. C. R. Matthews, L. N. Vaserstein and B. Weisfeiler, Congruence properties of Zariski dense subgroups, Proc. London Math. Soc. 48 (1984), pp. 514-532. MR735226 (85d:20040)

9. M. V. Nori, On subgroups of $G L_{n}\left(\mathbf{F}_{p}\right)$, Invent. Math. 88 (1987), pp. 257-276. MR880952 (88d:20068)

10. M. Suzuki, Group Theory I, Grundlehren der Mathematischen Wissenschaften, 247, SpringerVerlag (1982). MR648772 (82k:20001c)

11. B. Weisfeiler, Strong approximation for Zariski-dense subgroups of semisimple algebraic groups, Annals of Math. (2) 120 (1984), pp. 271-315. MR763908 (86m:20053)

12. B. Wehrfritz, Infinite Linear Groups, Ergebnisse der Matematik und ihrer Grenzgebiete, Band 76, Springer-Verlag (1973). MR0335656 (49:436)

Department of Mathematics, University of California, Santa Barbara, California 93106

E-mail address: cooper@math.ucsb.edu

Department of Mathematics, University of California, Santa Barbara, California 93106

E-mail address: long@math.ucsb.edu

Department of Mathematics, University of Tennessee, Knoxville, Tennessee 37996

E-mail address: morwen@math.utk.edu 